\title{
Caractérisation des boues de vidange dépotées sur les lits de séchage de zagtouli (Ouagadougou)
}

\author{
Martine $\mathrm{KONE}^{1 *}$, Emmanuelle SERVICE ${ }^{2}$, Yacouba OUATTARA ${ }^{1}$, Paul OUATTARA ${ }^{1}$, \\ Lucien $\mathrm{BONOU}^{3}$ et Pierre JOLY ${ }^{4}$
}

\author{
${ }^{1}$ Institut de Recherche en Sciences Appliquées et Technologies / CNRST, 03 BP 7047, Ouagadougou 03, \\ Burkina Faso. \\ ${ }^{2}$ Ecole Polytechnique Privée de la Jeunesse (ESUP-J). \\ ${ }^{3}$ UFR/SEA, Université de Ouagadougou, 03 BP 7021 Ouagadougou 03, Burkina Faso. \\ ${ }^{4}$ Laboratoire d'Écologie des Hydrosystèmes Naturels et Anthropisés, Université Claude Bernard Lyon 1, 43, \\ boulevard du 11 nov 1918, 69622 Villeurbanne, France. \\ *Auteur correspondant; E-mail : kbbamba20@yahoo.fr; koneba2003@yahoo.fr; Tel : +22670307668.
}

\section{REMERCIEMENTS}

Nous adressons nos vifs remerciements à l'Office National de l'Eau et de l'Assainissement du Burkina Faso pour l'accompagnement et les facilités accordées à l'équipe lors des sorties de terrain.

\section{RESUME}

A Ouagadougou comme dans la plupart des grandes villes africaines, il y a très peu de réseau d'égout. Les ouvrages d'assainissement autonome sont les plus couramment utilisés. Ces ouvrages produisent des boues qu'il faut vidanger et traiter adéquatement afin de préserver la santé des populations et protéger l'environnement. En effet, les risques sont réels, eu égard à l'utilisation des boues de vidange fraiches dans l'agriculture, en plus des dangers potentiels de leurs rejet à l'état brut dans la nature. A cet effet, le traitement par déshydratation constitue une solution. Les boues dépotées à la station de traitement par lits de séchage de Zagtouli (Ouagadougou) sont essentiellement issues des fosses septiques et des fosses toutes eaux. L'objectif du présent travail est de mettre en exergue les caractéristiques de ces boues pour guider le choix de technologies alternatives de traitement. A cet effet, les paramètres physico-chimiques et bactériologiques ont été mesurés selon les normes françaises. Les principaux résultats indiquent que la Demande Chimique en Oxygène (DCO) et la Demande Biochimique en Oxygène $\left(\mathrm{DBO}_{5}\right)$ de ces boues fluides, présentent des valeurs moyennes respectives de $1950 \mathrm{mg} \mathrm{O} / \mathrm{let} 785 \mathrm{mg} \mathrm{O} / \mathrm{l}$. Quant aux nutriments, ces boues se sont révélées particulièrement riches en azote ammoniacal avec une teneur moyenne de $557 \mathrm{mg} / \mathrm{l}$, tandis que les nitrates et orthophosphates ont des concentrations moyennes de 24 et $39 \mathrm{mg} / \mathrm{l}$ respectivement.

(C) 2016 International Formulae Group. All rights reserved.

Mots clés : Ouagadougou, lits de séchage, boues de vidange, $\mathrm{DBO}_{5}$, ammonium. 


\title{
Characterization of the waste sludge deposited on the Zagtouli drying bed (Ouagadougou)
}

\begin{abstract}
In Ouagadougou, as in most major African cities, there is very little sewerage. Autonomous systems are the most commonly used. These systems produce faecal sludge that must be drained and treated adequately in order to preserve the health of the population and protect the environment. Indeed, the risks are real, given the use of fresh sewage sludge in agriculture, in addition to the potential dangers of their raw rejection in nature. For this purpose, treatment by dehydration constitutes a solution. The faecal sludge deposited at the Zagtouli dry-bed treatment plant (Ouagadougou) is mainly from septic tanks and all-water pits. The goal of this study is to determine the characteristics of this faecal sludge for guiding the choice of alternative treatment technologies. Then, physical, chemical and bacteriological parameters were determined using French standards. The major results show that the Chemical Oxygen Demand (COD) and the Biochemical Oxygen Demand (BOD) of this fluid sludge have average values of $1950 \mathrm{mg} \mathrm{O}_{2} / 1$ and $785 \mathrm{mgO}_{2} / 1$ respectively. As for the nutrients, this faecal sludge was found to be particularly rich in ammoniacal nitrogen with an average content of $557 \mathrm{mg} / \mathrm{l}$, while nitrate and orthophosphate concentrations reached 24 and $39 \mathrm{mg} / \mathrm{l}$ respectively.

(C) 2016 International Formulae Group. All rights reserved.
\end{abstract}

Keywords: Ouagadougou, drying beds, faecal sludge, $\mathrm{BOD}_{5}$, ammonium.

\section{INTRODUCTION}

Dans les villes des pays en développement en général et celles d'Afrique Sub-Saharienne en particulier, les problèmes d'assainissement des eaux usées et excréta constituent un souci majeur (Coulibaly et al., 2004 ; Koné, 2011 ; Gnagne et al., 2015). Pour répondre au besoin d'ouvrage d'assainissement, le choix se porte préférentiellement sur les systèmes autonomes pour leurs coûts plus accessibles comparativement au réseau d'égout, et ce en dépit des risques sanitaires liés à la gestion des boues issues de ces ouvrages (Defo et al., 2015).

Selon l'Organisation Mondiale de la Santé (OMS), on dénombre dans le monde 2.6 milliards de personnes qui n'ont pas accès à des systèmes d'assainissement adéquats et près de 1.1 milliards de personnes continuent à déféquer en plein air (OMS- UNICEF, 2010).

S'agissant des pays en développement en particulier, un rapport des Nations Unies sur les Objectifs du Millénaire pour le Développement (OMD), estimait en 2008 que près d'un quart de la population des pays en développement ne disposait d'aucun système d'assainissement tandis que $15 \%$ utilisaient des installations sanitaires non adéquates. En outre, la défécation à l'air libre pratiquée par la majeure partie de la population met en danger l'ensemble de la communauté, eu égard aux risques accrus de contracter des maladies diarrhéiques, le choléra, des infestations vermineuses, l'hépatite et d'autres maladies associées (UN, 2008).

Cependant, au Burkina Faso, de 2005 à 2015 la politique d'assainissement a été orientée vers l'accroissement du taux d'accès à l'assainissement par la mise en place de ces ouvrages autonomes qui engendre d'importantes quantités de boues de vidange, avec son corollaire de gestion et dépotage anarchiques dans la ville et ses périphéries (Figure 1). Pour réduire les impacts sanitaires et écologiques de ces dépôts sauvages, deux stations de traitement des boues de vidange ont vu le jour à Kossodo et Zagtouli, des quartiers périphériques de Ouagadougou. Ces stations sont constituées de lits de séchage suivis de bassins de lagunage pour la prise en charge du lixiviat issu du traitement des boues.

La présente étude, a pour objectif d'étudier les caractéristiques des boues de vidange brutes dépotées à la station de traitement de Zagtouli, dans la perspective de proposer et expérimenter ultérieurement d'autres alternatives technologiques de traitement de ces boues, accessibles aux collectivités dans le contexte des pays en développement. 


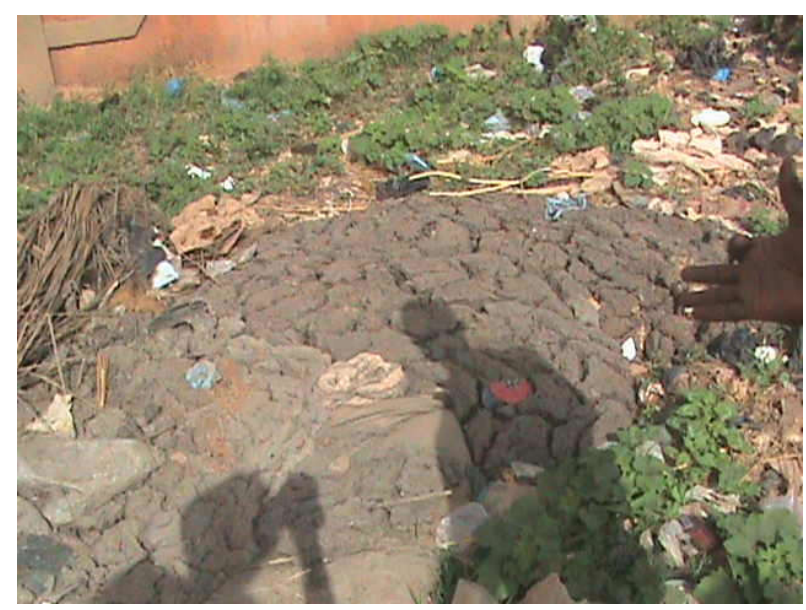

Figure 1 : Dépotage anarchique des boues de vidange.

\section{MATERIEL ET METHODES \\ Présentation du site d'étude}

La station de séchage des boues de Zagtouli est située à l'Ouest de la ville de Ouagadougou, non loin du péage de la route Nationale $\mathrm{N}^{\circ}$. Elle a été inaugurée en septembre 2014. Cette station a pour fonction le traitement des boues de vidange dans l'optique de sa valorisation dans l'agriculture. Elle comporte 48 lits de séchage qui mesurent chacun $16 \mathrm{~m}$ de long sur $8 \mathrm{~m}$ de large avec un volume utile de $64 \mathrm{~m}^{3}$.

A l'entrée de chaque lit de séchage est disposé un dégrilleur qui sert à empêcher l'entrée des déchets grossiers et encombrants (déchets solides), dans les bassins. Chaque lit de séchage est composé de deux couches différentes, dont la première est faite de graviers de diamètres compris entre 15 à 25 $(\mathrm{mm})$, avec une épaisseur de 20 à $30(\mathrm{~cm})$, et la deuxième couche de sable de $15(\mathrm{~cm})$ d'épaisseur. Ce traitement se fait de manière naturelle par l'infiltration, l'évaporation et la déshydratation grâce au vent et à l'énergie solaire. Le temps de séchage de ces boues peut varier de deux à trois semaines. Les lixiviats issus de la filtration sont drainés dans une petite station d'épuration des eaux usées par lagunage composées d'une succession de trois bassins (un bassin anaérobie, un bassin facultatif et un bassin de maturation) pour un autre traitement avant leur rejet dans la nature.

\section{Matériel \\ Les boues de vidange}

Les boues de vidange désignent des boues fraîches ou partiellement minéralisées liquides ou solides résultant du stockage des eaux vannes et des excréta (Tilleyet al., 2008) dans des ouvrages non raccordés au réseau d'égout. Il s'agit de: latrine traditionnelle, latrine VIP (Ventilated Improved Pit), latrine à chasse manuelle, toilette publique, puisard, fosse septique et de fosse toutes eaux. Selon le type d'ouvrage, la consistance et la composition des boues sont variables. En effet, les boues issues des ouvrages à remplissage rapide et utilisant peu d'eau tels que les latrines traditionnelles, les latrines VIP et les toilettes publiques sont très concentrées et fermentescibles, tandis que les boues provenant des fosses septiques après un séjour plus ou moins long de 4 à 15 ans (Defo et al., 2015) sont partiellement minéralisées au cours de processus anaérobies de digestion; elles sont biologiquement plus stables. Il existe deux modes de vidange au Burkina, à savoir la vidange manuelle qui concerne essentiellement les latrines (Figure 2) et la vidange mécanique 
dans le cas des fosses septiques et fosses toutes eaux (Figure 3). Dans le premier cas, il s'agit de boues concentrées et dans le second cas elles sont diluées, ce qui facilite la vidange. En effet, Defo et al. (2015) rapportent que la fosse septique est un ouvrage moderne de prétraitement (décantation et digestion) des boues de vidange ce qui justifie que les concentrations en polluants soient moins élevées qu'au niveau des latrines.

\section{Matériels de prélèvements et équipements de protection}

Le matériel de prélèvement est composé d'un gobelet, d'une glacière, d'un entonnoir et de flacons (verre et plastique) tandis que le matériel de protection individuelle est constitué de gants à usage multiple, de gants à usage unique, de cache nez, de blouse et des chaussures de sécurité.

\section{Matériel de conditionnement et de conservation}

Des flacons en plastique d'une capacité d'un litre contenant les échantillons destinés aux analyses physico-chimiques et des flacons en verre de $500 \mathrm{ml}$ stérilisés destinés aux analyses bactériologiques, ont été utilisés. Chaque flacon de prélèvement porte des indications relatives au nom du préleveur, la date, l'heure, le lieu de prélèvement, et les valeurs des paramètres relevés in situ conformément aux normes en vigueur. Les échantillons sont placés dans une glacière et transportés jusqu'au laboratoire où ils sont conservés dans un réfrigérateur à la température maximale de $4{ }^{\circ} \mathrm{C}$ après ajout de réactifs de conservation au besoin.

\section{Méthodes d'analyse des échantillons}

Aux cours de ce travail les échantillons de boues brutes dépotées fraichement ont été prélevés juste après le remplissage dans différents lits (Figure 3) désignés par B1, B2, $\mathrm{B} 3$ et $\mathrm{B} 4$ à raison d'un lit par mois. Il est à noter que les boues ont été remuées par le préleveur avant le prélèvement afin d'avoir un échantillon homogène.

La température, le Potentiel Hydrogène $(\mathrm{pH})$, l'oxygène dissous $\left(\mathrm{O}_{2}\right.$ dissous) et la Conductivité Electrique (CE) ont été mesuréesin situ avec le Multi paramètres HACH HQ40d muni des différentes sondes, suivant le même protocole en changeant simplement de sondes en fonction du paramètre à mesurer. La lecture est faite après stabilisation de l'affichage. La méthode est conforme à la norme AFNOR 90008 . La détermination des teneurs en Matières En Suspension (MES) a été faite par gravimétrie après filtration sous vide avec un filtre à microfibre de verre $\mathrm{GF} / \mathrm{C}$ et séchage à l'étuve à $105 \pm 2{ }^{\circ} \mathrm{C}$, conformément à la norme française NF 90-105. La Demande Chimique en Oxygène (DCO) est déterminée par oxydation avec un excès de dichromate de potassium en milieu acide à $150{ }^{\circ} \mathrm{C}$, en présence de sulfate d'argent comme catalyseur et de sulfate de mercure destiné à éviter l'interférence de la présence d'ions chlorure, conformément à la norme AFNOR T国900101. La lecture de la valeur est faite par spectrophotométrie $\mathrm{HACH}$ $\mathrm{DR} / 2400$ à la longueur d'onde adéquate selon la gamme de DCO choisie. La Demande Biochimique en Oxygène de cinq jours $\left(\mathrm{DBO}_{5}\right)$ a été déterminée par la méthode d'incubation pendant cinq jours à l'obscurité et à la température de $20^{\circ} \mathrm{C}$ à l'aide d'un appareil "LovibondOxiDirect", la mesure est faite par des sondes de pression électroniques résistantes selon la norme NF 900103. L'azote ammoniacal $\left(\mathrm{NH}_{4}{ }^{+}\right)$, les nitrates $\left(\mathrm{NO}_{3}{ }^{-}\right.$ ), les nitrites $\left(\mathrm{NO}_{2}-\right)$ et les orthophosphates $\left(\mathrm{PO}_{4}{ }^{3+}\right)$ ont été déterminés par la méthode colorimétrique avec une lecture au spectrophotomètre Palintest 7100, aux longueurs d'onde adéquates. S'agissant des Coliformes Fécaux (CF) et des Streptocoques Fécaux (SF), la méthode est basée sur la recherche et le dénombrement des colonies de bactéries par la technique de filtration sur membrane et ensemencement dans des milieux de culture appropriés (gélose spécifique chromocult Agar pour les CF et gélose spécifique chromocult Agar pour les SF) suivie de l'incubation à la température adéquate pendant 18 à $24 \mathrm{~h}$. La présente méthode est conforme à la norme française NF EN ISO 9308-1 (Septembre2000). L'analyse des données a été faite sur Excel. 


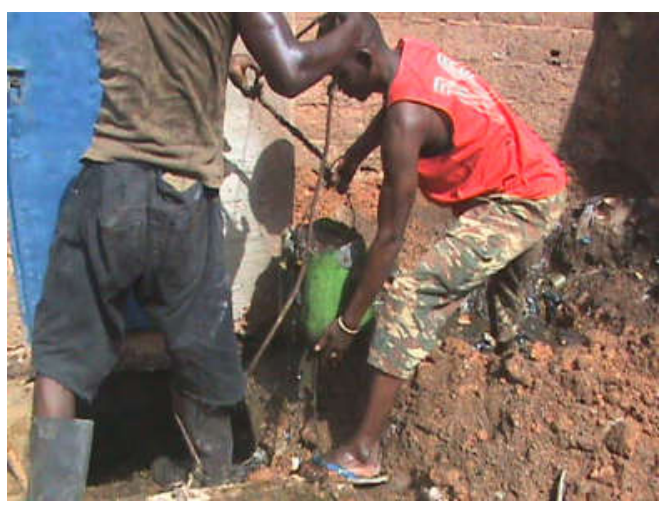

Figure 2 : Vidange manuelle d'une latrine familiale.

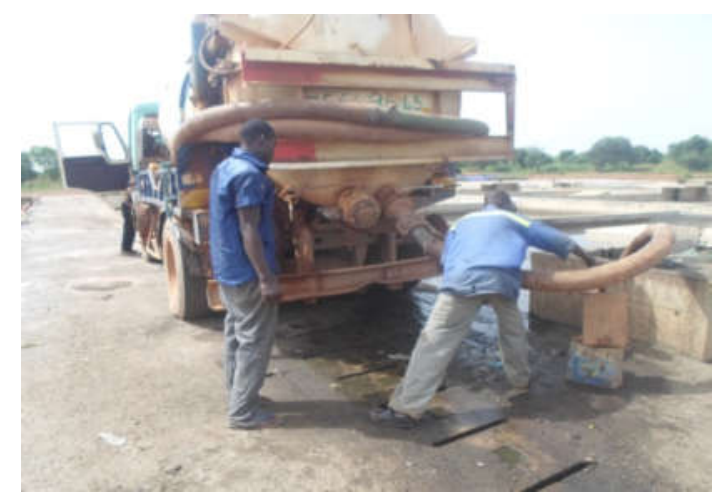

Figure 3: vidange mécanique sur les lits de séchage.

\section{RESULTATS}

\section{Provenance et caractéristiques des boues de vidange}

La station de Zagtouli reçoit des boues provenant essentiellement de fosses septiques, de fosses toutes eaux et de puisards, vidangés par des camions citernes de capacités variant entre $3,5 \mathrm{~m}^{3}$ et $16 \mathrm{~m}^{3}$. Le choix quasi exclusif de ces ouvrages s'explique par la difficulté de vidange mécanique des latrines qui reçoivent peu d'eau, alors qu'elles sont les plus répandues dans la ville.

Compte tenu de l'origine des boues (fosses septiques, fosses toutes eaux et puisards), celles-ci sont très diluées, justifiant ainsi le traitement du lixiviat par lagunage à l'aval des lits.

\section{Caractéristiques physico-chimiques des boues}

Le Tableau 1 présente les différents paramètres de pollution relevés entre juillet et octobre 2015 au niveau de quatre lits de séchage, aussitôt après le remplissage des bassins. En comparant les valeurs moyennes des paramètres avec les normes de rejets dans la nature, il s'avère que les paramètres physiques comme la température (Figure 4), le pH (Figure 5 ) et les teneurs en nitrites (Figure 11) sont conforment aux normes de rejet fixées au Burkina Faso. Les teneurs en oxygène dissous restent faibles puisqu'elles ont varié entre 0,17 et $0,35 \mathrm{mg} / 1$ (Figure 7). Les teneurs des matières en suspensions (MES) a varié entre 500 et 2000 $\mathrm{mg} / \mathrm{l}$ au sein des différents bassins, alors que la norme autorise une valeur limite de $150 \mathrm{mg} / \mathrm{l}$ (Figure 8).

Quant aux nutriments précurseurs d'eutrophisation des plans d'eau, les concentrations en ammonium (Figure 9) dépassent largement la limite exigée de $35 \mathrm{mg} / \mathrm{l}$ pour l'ensemble des formes oxydables (c'est-àdire azote organique et ammonium réunis); Il en est de même pour les nitrates avec des 
teneurs variant entre 14,4 et 30,4 mg / (Figure 10). Ces valeurs ne sont pas conformes aux objectifs de qualité qui fixent la limite à 11,4 $\mathrm{mg} / 1$; Les ortho-phosphates, faisant parti des nutriments présentent des teneurs excédant largement la valeur limite admise par la loi qui est de $0,8 \mathrm{mg} / 1$, puisque les teneurs ont oscillé entre 13,7 et 60,2 mg/l (Figure 12). Au vu de ces résultats, il s'avère que les teneurs en nitrates et ortho-phosphates qui sont des substances indésirables, parce que source d'eutrophisation (Nyenje et al., 2010 ; Tapsoba et al., 2016) dépassent largement les normes de rejet fixées par la règlementation du Burkina Faso.

S'agissant de la pollution carbonée, la Demande Chimique en Oxygène (DCO) et la Demande Biochimique en Oxygène de cinq jours $\left(\mathrm{DBO}_{5}\right)$ ont donnée des valeurs variant respectivement entre 1400 et $2760 \mathrm{mg} / \mathrm{l} \mathrm{d}$ 'une part et 649 et $980 \mathrm{mg} / \mathrm{l}$ (Figure 13) d'autre part ; Même si ces valeurs sont relativement très faibles comparativement à celles relevées par d'autres auteurs, elles restent largement supérieures aux normes qui imposent respectivement 150 et $40 \mathrm{mg} / \mathrm{l}$ pour ces paramètres.

Il convient de noter que si la température, le $\mathrm{pH}$ et la teneur en oxygène dissous varient peu d'un bassin à l'autre, les paramètres tels que la conductivité, la $\mathrm{DCO}$, la $\mathrm{DBO}_{5}$, les MES d'une part, ou l'azote et le phosphore d'autre part, présentent des valeurs aléatoires, renvoyant ainsi à l'origine des boues dépotées dans les bassins. Dans tous les cas, les valeurs restent largement supérieures aux normes admises au plan national pour le rejet dans la nature ou pour la réutilisation en agriculture (WHO, 2006). Cet état de fait conforte la nécessité de traiter ces boues de façon adéquate, afin de réduire les risques sanitaires et environnementaux (Koné et Strauss, 2004).

\section{Caractéristiques bactériologiques des boues}

Les résultats des analyses bactériologiques sont consignés dans le Tableau 2. Il ressort que les teneurs moyennes en $\mathrm{CF}$ et $\mathrm{SF}$ sont similaires et faibles comparativement aux valeurs relevées par d'autres auteurs. En effet, les teneurs en pathogènes dépendent de facteurs tels que la durée de stockage dans l'ouvrage, la température du milieu et le taux de dilution.

Defo et al. (2015) rapportent des teneurs en SF atteignant $100 \mathrm{UFC} / 100 \mathrm{ml}$ et jusqu'à $10000 \mathrm{UFC} / 100 \mathrm{ml}$ de $\mathrm{CF}$ dans les eaux de puits traduisant ainsi la vulnérabilité des ressources en eau au regard de la pollution bactériologique liée aux infiltrations issues des latrines.

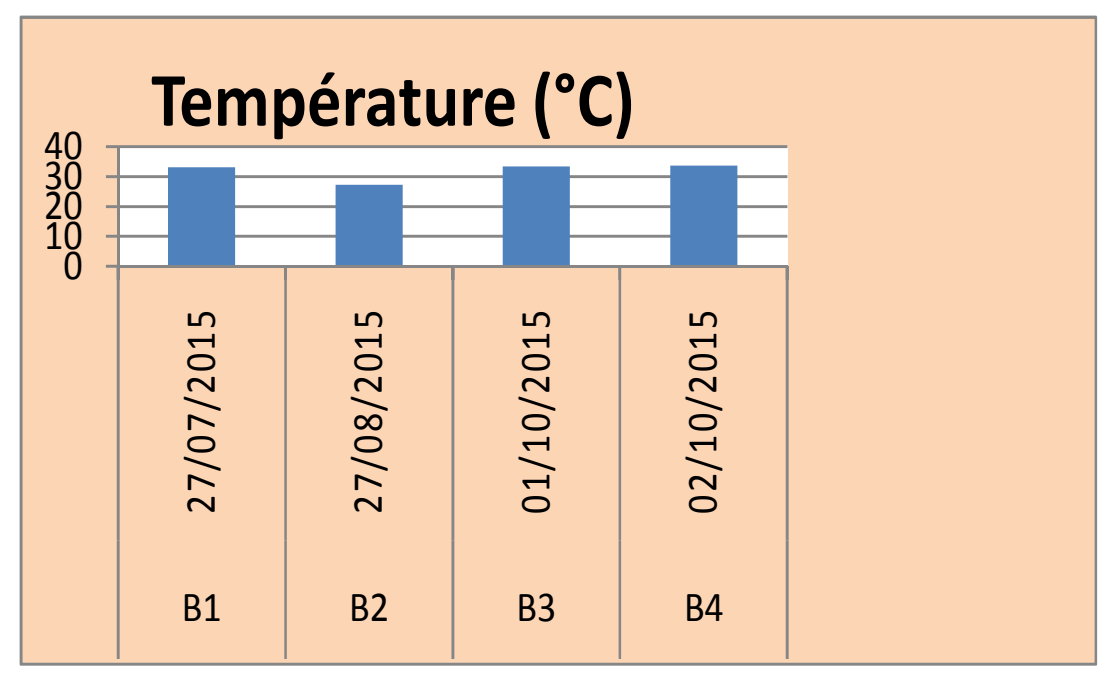

Figure 4: Evolution de température dans les différents lits de séchage. 
Tableau 1 : Caractéristiques physico-chimiques des boues de vidange brutes dépotées à la station de Zagtouli.

\begin{tabular}{|c|c|c|c|c|c|c|}
\hline Dates & $07 / 15$ & $08 / 15$ & $9 / 15$ & $10 / 15$ & Moyenne & $\begin{array}{l}\text { Normes de } \\
\text { rejets (Burkina } \\
\text { Faso, 2015) }\end{array}$ \\
\hline Bassins & B1 & B2 & B3 & B4 & & \\
\hline $\mathbf{T}\left({ }^{\circ} \mathrm{C}\right)$ & 33 & 27,2 & 33,5 & 33,6 & 31,82 & $18-40$ \\
\hline pH & 7,45 & 7,44 & 7,75 & 7,7 & 7,58 & $6,5-9$ \\
\hline CE (uS/cm) & 4350 & 2079 & 2690 & 4450 & 3392 & 1000 \\
\hline $\mathrm{O}_{2}$ dissous $\left.\mathrm{mg} / \mathrm{l}\right)$ & 0,21 & 0,35 & 0,17 & 0,21 & 0,235 & \\
\hline MES (mg/l) & 1000 & 500 & 2000 & 1000 & 1125 & 150 \\
\hline $\mathrm{NH}_{4}(\mathrm{mg} / \mathrm{l})$ & & 130 & 1030 & 615 & 557 & 35 \\
\hline $\mathrm{NO}_{3}(\mathrm{mg} / \mathrm{l})$ & 29 & 20,9 & 30,4 & 14,4 & 23,7 & 11,4 \\
\hline $\mathrm{NO}_{2}(\mathrm{mg} / \mathrm{l})$ & 0,094 & 0,03 & 0 & 0,07 & 0,049 & 0,9 \\
\hline $\mathrm{PO}_{4} / \mathrm{l}(\mathrm{mg} / \mathrm{l})$ & 13,7 & 29 & 55 & 60,2 & 39,5 & 0,8 \\
\hline $\mathrm{DCO}\left(\mathrm{mgO}_{2} / \mathrm{l}\right)$ & 1400 & 2000 & 2760 & 1640 & 1950 & 150 \\
\hline $\mathrm{DBO}_{5}\left(\mathrm{mgO}_{2} / \mathrm{l}\right)$ & 980 & 649 & 711 & 800 & 785 & 40 \\
\hline
\end{tabular}

Tableau 2 : Comparaison des valeurs des paramètres bactériologiques obtenues par rapport à celles d'autres villes.

\begin{tabular}{llllll}
\hline Paramètre & $\begin{array}{l}\text { Ouagadougou } \\
\text { (Zagtouli) }\end{array}$ & $\begin{array}{l}\text { Baro 2012 } \\
\text { Jahara }\end{array}$ & Almuzan-2008 & $\begin{array}{l}\text { Radahaidah and al } \\
\text { Zboon (2011) } \\
\text { Jordanie }\end{array}$ & \\
\hline CF (UFC/100ml) & $3,2.10^{5}$ & $3,01.10^{5}$ & $4,62.10^{8}$ & $4,6.10^{8}$ \\
\hline SF (UFC/100ml) & $3,08.10^{5}$ & $2,2.10^{6}$ & & & \\
\hline
\end{tabular}

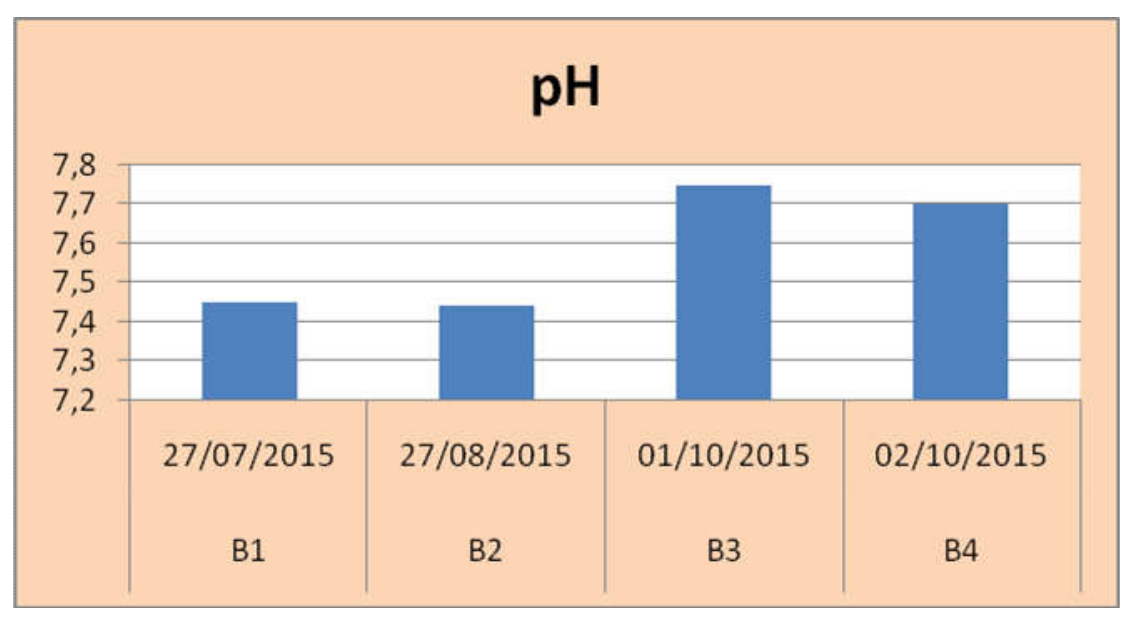

Figure 5: Evolution de $\mathrm{pH}$ dans les différents lits de séchage. 


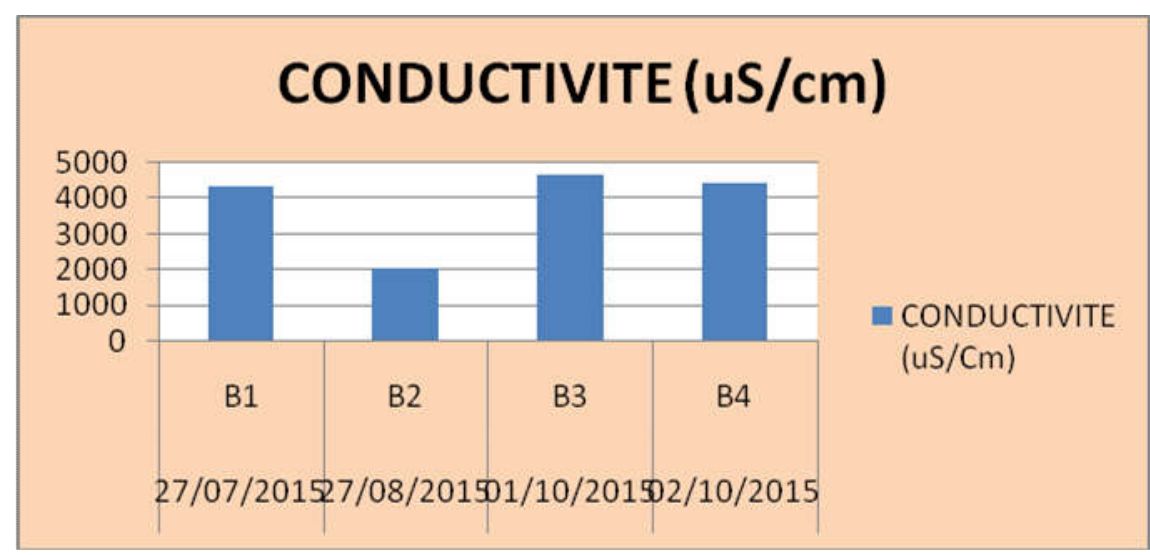

Figure 6 : Evolution de la conductivité des boues dans les différents lits.

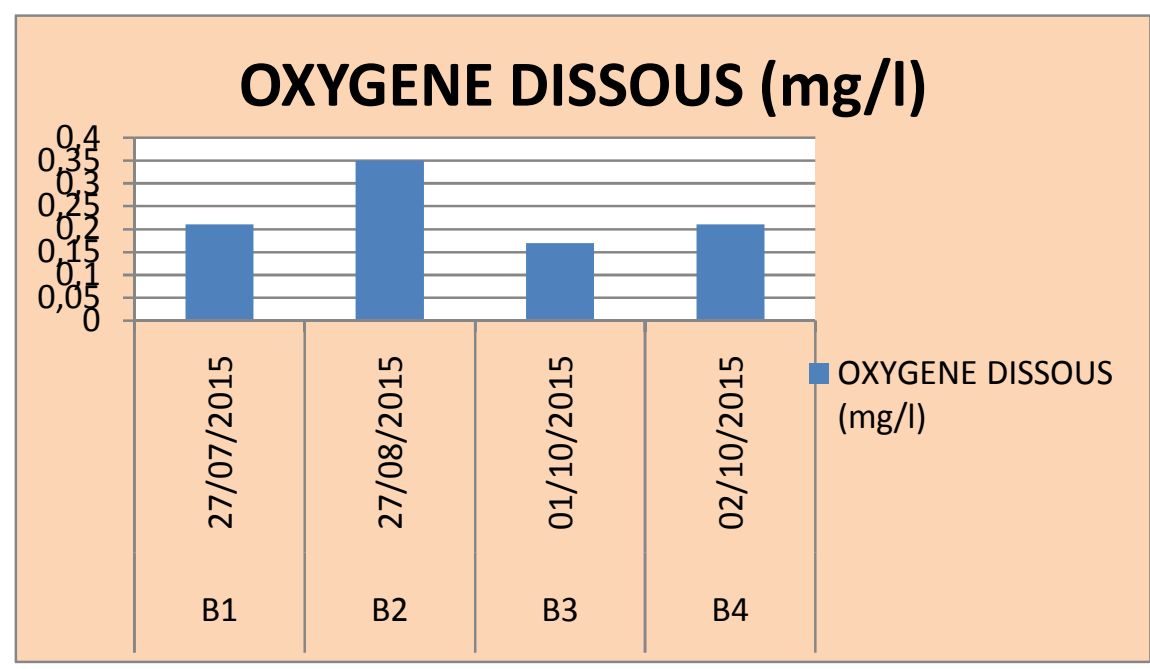

Figure 7 : Evolution des teneurs en oxygène dissous au niveau des différents lits.

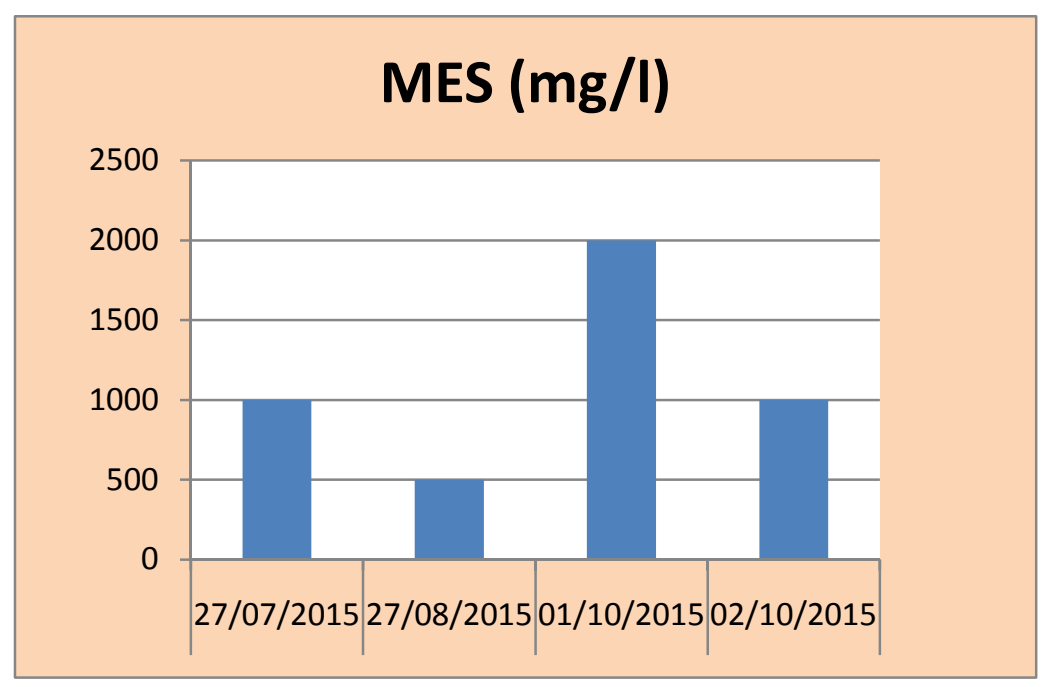

Figure 8: Evolution des teneurs en MES dans les différents lits de séchage. 


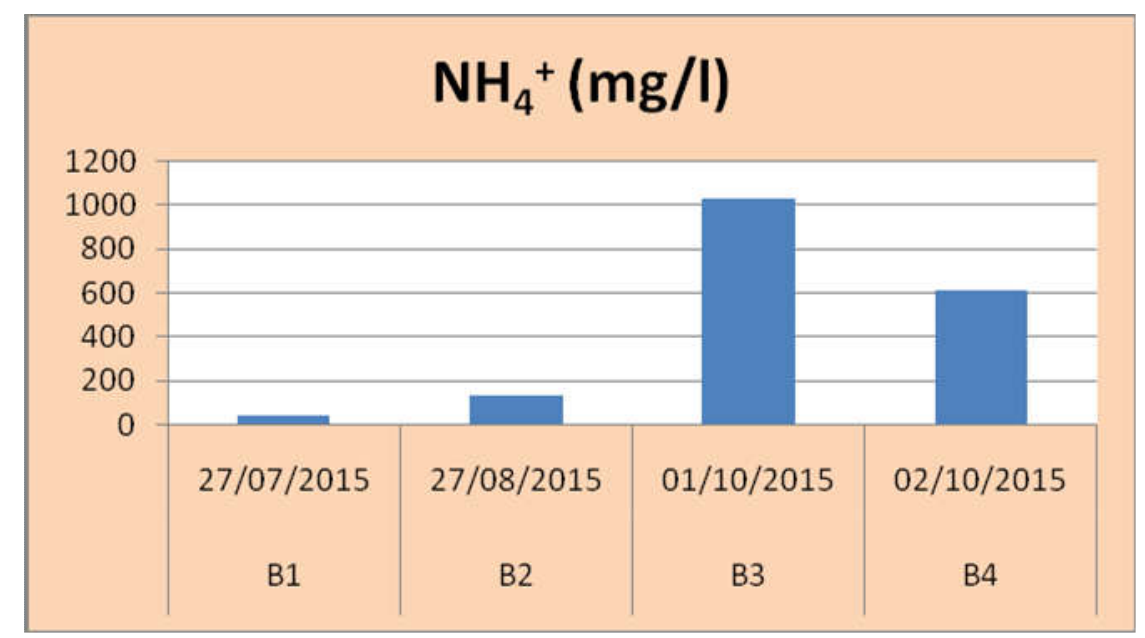

Figure 9: Evolution de teneurs en ammonium $\mathrm{NH}_{4}{ }^{+}$dans les différents lits.

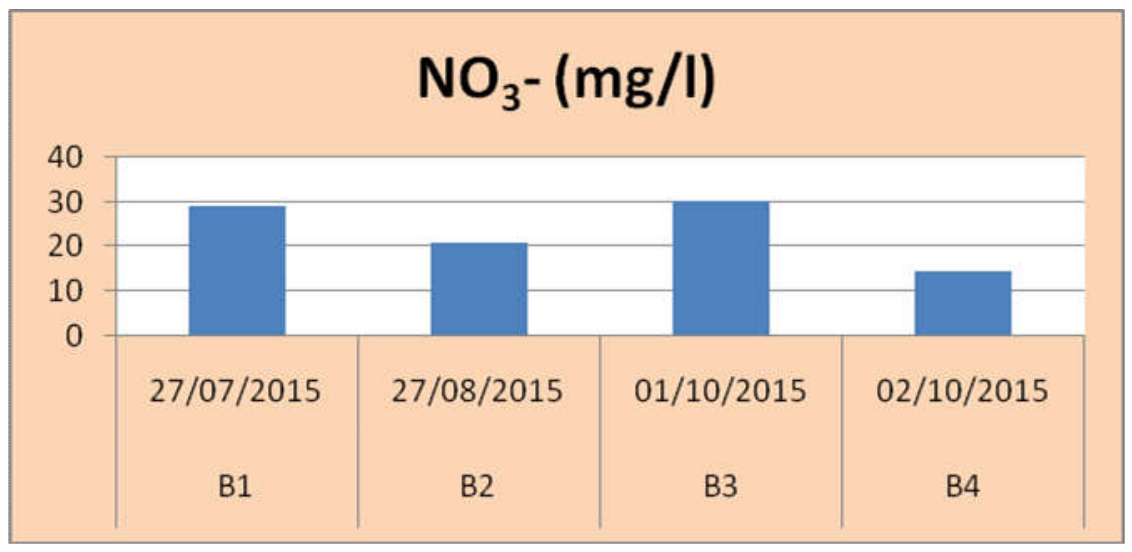

Figure 10 : Evolution des teneurs en nitrates $\mathrm{NO}_{3}{ }^{-}$dans les différents lits de séchage.

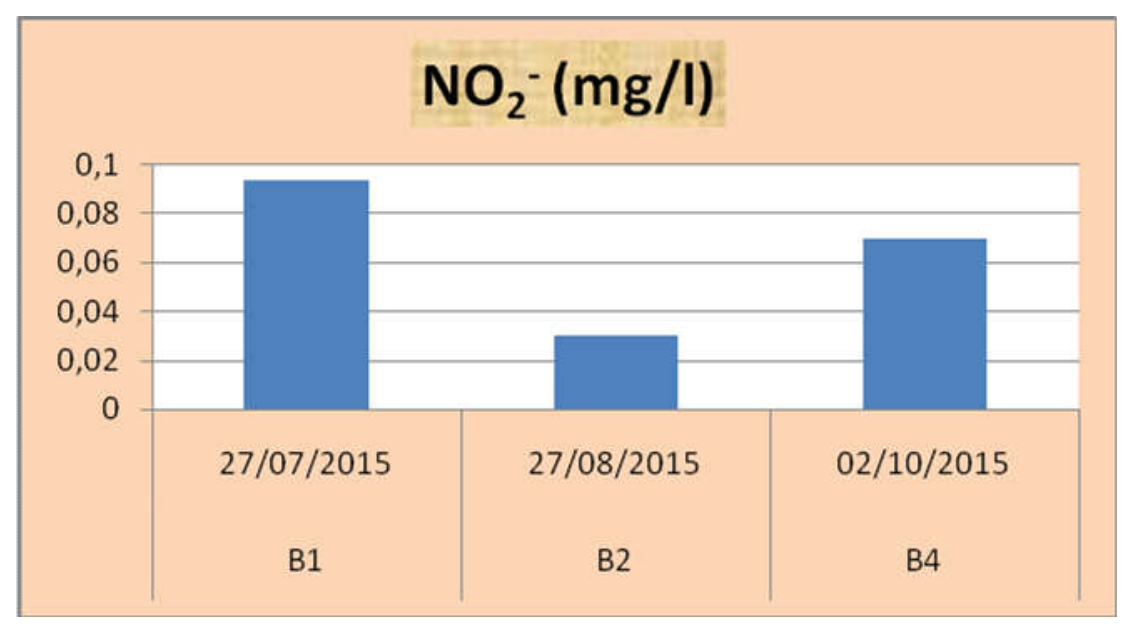

Figure 11 : Evolution des teneurs en nitrites $\mathrm{NO}_{2}^{-}$dans les bassins. 


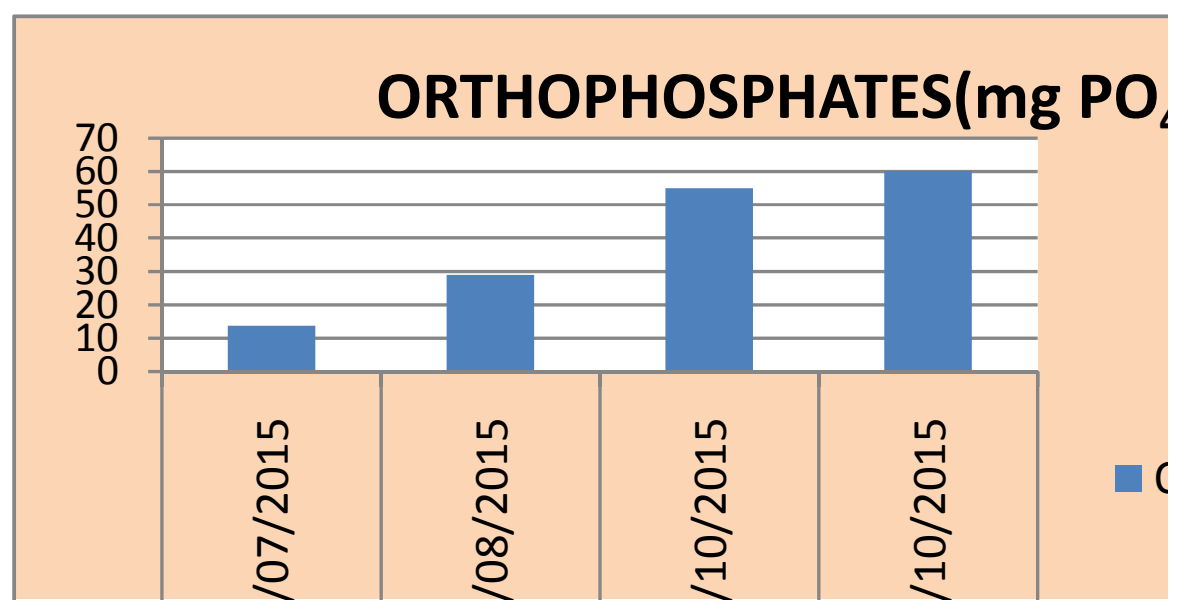

Figure 12: Diagramme d'évolution des teneurs en orthophosphates.

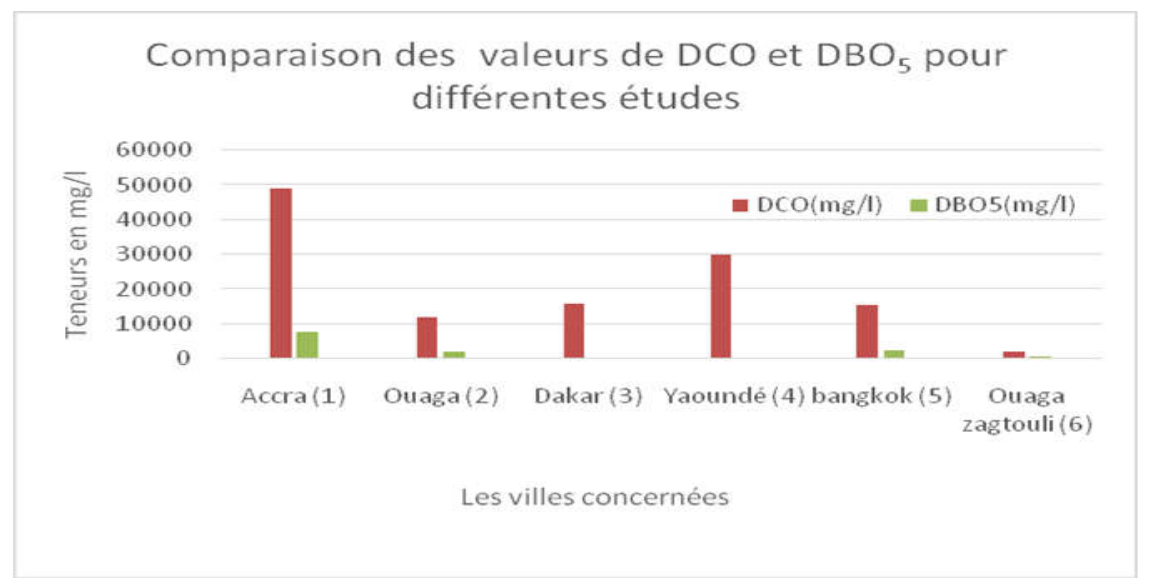

Figure 13 : Courbe de comparaison de quelques paramètres pour différentes villes. [1]Koné et Strauss, 2004; [2]Mahamane, 2011; [3]Walker, 2008; [4] Kengne, 2006; [5]Koottatep et al,.2005; [6] La présente étude.

DISCUSSION

\section{Paramètre physico-chimiques Température}

Les températures maximale et minimale enregistrées au cours de la période de l'étude (juillet-octobre) sont respectivement $33,6^{\circ} \mathrm{C}$ et $27,2{ }^{\circ} \mathrm{C}$. La Figure 4 indique la variation de la température suivant les bassins. Les températures relevées au mois d'octobre dans les bassins B1, B3 et B4 sont plus élevées tandis que celle dans le bassin B2 est la plus faible. B2 correspond à la période hivernale avec une baisse sensible des températures. Il faut noter que la température est favorable à l'activité microbienne de minéralisation au sein des bassins. En effet, pour évaluer les caractéristiques d'une station de traitement, la température est un paramètre non négligeable. Sa variation brusque, tout comme celle du $\mathrm{pH}$ à des effets considérables sur le comportement des bactéries épuratrices du milieu. Ainsi on note qu'en période chaude l'activité biologique est plus importante qu'en hiver, car celle-ci joue un rôle majeur sur la cinétique des réactions.

\section{Potentiel hydrogène $\mathrm{pH}$}

Lors de cette étude, au niveau de la station de traitement de Zagtouli, la valeur moyenne du $\mathrm{pH}$ tout en étant supérieure à $7 \mathrm{n}$ 'a 
atteint 8 au niveau d'aucun bassin. Les valeurs relevées restent conformes à la norme qui impose 6,5 à 9. Comme l'indique la Figure 5, les $\mathrm{pH}$ extrêmes obtenus sont respectivement 7,44 et 7,75 , des valeurs compatibles avec le développement des bactéries qui assurent l'épuration des boues. En effet, le $\mathrm{pH}$, la température et la salinité peuvent être des facteurs limitants pour le développement des bactéries épuratrices. Celles-ci se développent en général à des $\mathrm{pH}$ compris entre 5 et 9 . A titre d'exemple les bactéries de la nitritation telles que Nitrosomonas se multiplient dans un milieu alcalin avec un $\mathrm{pH}$ compris entre 7,4 et 9 tandis que celles de la nitratation croissent dans un milieu dont le $\mathrm{pH}$ se situe entre 8,5 et 9,1 . C'est pourquoi ces différents paramètres peuvent limiter la croissance de certaines bactéries impactant ainsi l'efficacité du traitement (Degrémont, 2005).

\section{Conductivité}

La conductivité au niveau des différents bassins a varié entre 2079 et $4450 \mu \mathrm{S} / \mathrm{cm}$ comme le montrent le Tableau1 et la Figure 6. Il ressort que les plus fortes valeurs ont été prises au niveau du bassin B1 et B4. Ceci indique que les boues dépotées à la station de Zagtouli sont très minéralisées, mais restent toujours fermentescibles eu égard aux teneurs en polluants organiques biodégradables. Ces résultats confirment qu'il y'a une forte présence de sels minéraux dans le milieu; tenant compte des risques de contamination des eaux de surface, une augmentation excessive de la conductivité des milieux aquatiques engendre des modifications de l'écosystème bactérien et a une influence également sur la survie de la faune et de la flore aquatiques. En outre, une salinité trop importante provoque le colmatage du sol (Koné et al., 2012), et impacte négativement l'évolution de bon nombre de spéculations en agriculture. A titre d'exemple la présence excessive de sels provoque un disfonctionnement des pompes ioniques permettant à la plante, en temps normal d'absorber cet ion.

\section{Oxygène dissous}

Les teneurs en oxygène dissous obtenues au niveau des bassins concernés sont faibles et sont comprises entre $0,17 \mathrm{mg} / 1$ et 0,35 $\mathrm{mg} / \mathrm{l}$ (Figure 7). Ceci pourrait s'expliquer par le fait que les boues proviennent d'ouvrages où règnent des processus anaérobies d'une part et d'autre part par l'activité photosynthétique des algues encore très faible puisque les bassins viennent d'être remplis, en sus du fait que la présence importante de matière en suspension ne favorise pas le processus photosynthétique. Par ailleurs, le phénomène de transfert d'oxygène à l'interface air-surface des boues contribue faiblement à ce résultat.

\section{Matières en suspension (MES)}

La concentration des matières en suspensions (MES) est très élevée dans les boues. Comme l'indique la Figure 8, les teneurs ont varié entre 500 et $2000 \mathrm{mg} / \mathrm{l}$ avec une moyenne de $1125 \mathrm{mg} / \mathrm{l}$. Ces valeurs dépassent largement la norme indiquée qui est de 150 $\mathrm{mg} / \mathrm{l}$. Certains auteurs ont relevé des teneurs en MES plus importantes lors de travaux similaires. En effet, Mahamane (2011) fait état de valeur moyenne en MES de $11084 \mathrm{mg} / \mathrm{l}$ à Ouagadougou. Cela confirme la grande variabilité des caractéristiques des boues au regard de leur provenance. Les valeurs relativement faibles relevées pour cette étude, pourraient s'expliquer par la dilution au sein des ouvrages, mais aussi du fait des pluies et des conditions de décantation idéales. L'impact des eaux de pluie n'est pas négligeable non plus, étant donné que la plus faible valeur qui est de $500 \mathrm{mg} / \mathrm{l}$ a été relevée pendant la période correspondant aux fortes précipitations, même s'il reste entendu que la variabilité de la qualité des boues est aussi inhérente à leur provenance (Strauss and Koné, 2003).

Il faut relever que les matières en suspension (particulaires ou colloïdales) sont constituées de matières organiques, de micropolluants (organiques et minéraux), de micro-organismes (bactéries, virus...), de sels minéraux... Elles peuvent donc être sources de colmatage organique des filtres par obstruction 
des pores lors de l'infiltration du lixiviat (Koné, 2011), un phénomène qui a été constaté sur différents lits de la station à certains moments.

\section{Ammonium}

La dégradation de l'azote organique en ammonium fait partie intégrante du cycle de l'azote dans la production des nutriments. Des teneurs élevées en ammonium ont été relevées au niveau de tous les bassins (Figure 9); ce phénomène est d'autant plus prononcé que le milieu est alcalin. Ces teneurs en ammonium pourraient s'expliquer par la qualité de l'ouvrage (fosse septique ou latrine) et la fréquence de vidange. En effet, dans ces ouvrages les conditions d'anaérobiose favorisent la production d'ammonium, qui s'oxydera dans les conditions aérobies des lits de séchage. Par ailleurs, plus le séjour des boues dans la fosse est long, plus la minéralisation est importante. Les boues provenant des fosses septiques ne sont que partiellement minéralisées au moment de la vidange. La minéralisation est plus ou moins importante en fonction de la durée du stockage.

\section{Nitrates}

La concentration moyenne en nitrates est de $23,67 \mathrm{mg} / 1$ pour l'ensemble des bassins concernés, avec cependant, des teneurs atteignant 30,4 mg/l dans le bassin B3 (Figure 10). Cela dénote d'un bon processus de nitrification lors de la déshydratation des boues. Les valeurs relevées ne respectent pas la norme de rejet, justifiant ainsi de la nécessité d'une gestion du luxiviat, afin d'éviter le risque de pollution des eaux de surface et des eaux souterraines (Josse et al., 2016 ; Nyenje et al., 2010; Bricha et al., 2007; Rajmohan et Elango, 2005 ). A cet effet, à l'aval des lits de séchage de boues, une mini station de lagunage à microphytes reçoit le percolât issu de la filtration des boues. En outre, les teneurs en nitrates pourraient augmenter au fil du temps dans le lixiviat, eu égard au fait qu'il s'agit d'un processus aérobie.

\section{Nitrites}

Les ions nitrite, forme oxydée de l'azote, ne sont pas stables; ils évoluent en nitrates en milieu aérobie tel que c'est le cas dans le processus de déshydratation sur lit de séchage. La Figure 11 montre que les teneurs relevées restent inférieures aux objectifs de qualité imposés par le législateur qui est de 0,9 $\mathrm{mg} / \mathrm{l}$. Cela est favorisé par la bonne condition de nitrification, corroborée par la Figure 10 précédente, et confirme l'instabilité de cette forme de l'azote qui évolue instantanément en nitrate.

\section{Orthophosphates}

Les ortho-phosphates sont indésirables lorsque leurs teneurs deviennent trop importantes, bien que ce soit des nutriments pouvant être mis à profit dans l'agriculture. En effet, phosphore et azote contribuent à l'eutrophisation des eaux de surface, un phénomène qui porte préjudice à la flore et à la faune aquatiques (Nyenje et al., 2010; Tapsoba et al., 2016).

La Figure 12 qui donne les teneurs en orthophosphates dans les différents bassins indique que la concentration en orthophosphates varie de façon importante d'un lit à l'autre. Le phosphore qui se trouve dans les boues sous différentes formes (phosphore organique, orthophosphates...) peut être issue de l'alimentation mais également de sources naturelles ou d'activités anthropiques. Les concentrations particulièrement élevées et variant de $13,7 \mathrm{mg} / \mathrm{l}$ à $60,2 \mathrm{mg} / \mathrm{l}$ sont supérieures à la norme admise $(0,8 \mathrm{mg} / \mathrm{l})$. Cela s'explique par le fait que le traitement élimine très peu le phosphore qui reste adsorbé et piégé dans la matière en suspension en décantation; cependant, une partie des orthophosphates, sous la forme dissoute se retrouve dans le lixiviat.

\section{La pollution carbonée : $\mathrm{DBO}_{5}$ et DCO}

Les résultats d'analyses révèlent que le rapport des moyennes de la $\mathrm{DCO}$ et de la $\mathrm{DBO}_{5}$, donne 2,48 dénotant ainsi le caractère biodégradable des boues de vidange et le choix de la technologie biologique de traitement appliquée. En effet pour un rapport inférieur à 2,4 les boues sont toujours biodégradables et la minéralisation se poursuit (Degrémont, 2005). Ainsi, la matière organique formée essentiellement de glucides, de lipides et de protéines subira une dégradation spontanée en 
milieu aérobie ou en milieu anaérobie par l'action de microorganismes qui s'y développent, principalement des bactéries autotrophes ou hétérotrophes. Ces bactéries transforment la pollution organique carbonée biodégradable $\left(\mathrm{DBO}_{5}\right)$ et la pollution inorganique pour assurer leur multiplication et leur développement. A l'inverse, si le rapport $\mathrm{DCO} / \mathrm{DBO}_{5}$ est élevé, cela indique que les boues sont minéralisées et ne sont plus fermentescibles. Dans ce contexte un traitement biologique n'aura pas d'intérêt. Les valeurs moyennes de $\mathrm{DBO}_{5}$ et $\mathrm{DCO}$ respectivement de $1950 \quad \mathrm{mg} \quad \mathrm{O}_{2} / 1$ et $785 \quad \mathrm{mg} \quad \mathrm{O}_{2} / 1$ dépassent largement les normes de rejet. Si ces valeurs semblent élevées, certains auteurs ont fait état de teneurs plus importantes comme indiquées sur le graphique (Figure13). Les teneurs de $\mathrm{DCO}$ et $\mathrm{DBO}_{5}$ des boues de la station de Zagtouli relativement faibles pourraient s'expliquées par la grande dilution de ces boues. Cependant, même si les valeurs semblent faibles comparativement aux valeurs relevées par d'autres auteurs à Ouagadougou, Accra, Dakar, Yaoundé, (Kengné, 2006 ; Walker, 2008 ; Kottatep et al., 2005; Koné et Strauss, 2004, ; Mahamane, 2011), il faut noter que ces boues restent fermentescibles, ce qui justifie la nécessité de leur traitement et de celui du lixiviat avant rejet aux fins de les rendre conformes aux normes de rejet. Les résultats du présent travail et ceux obtenus par ces auteurs montrent la grande variabilité des caractéristiques des boues de vidange en fonction des pays (niveau de vie, habitudes alimentaires...) en plus du type d'ouvrage et du temps de séjour.

\section{La pollution microbiologique}

L'analyse bactériologique a porté sur la détermination des concentrations en Coliformes Fécaux et en Streptocoques Fécaux dans les boues brutes dépotées fraichement à la station. Les coliformes fécaux sont un groupe de bactéries faisant partie des coliformes totaux, mais incubés à une température plus élevée $\left(44,5^{\circ} \mathrm{C}\right)$ de façon à privilégier la croissance de ces bactéries et des streptocoques fécaux qui sont considérés comme des indicateurs d'une contamination d'origine fécale ancienne. Leur présence dans ces lits de séchage indique que ces boues sont susceptibles de contenir d'autres microorganismes pathogènes. Les coliformes fécaux constituent un groupe de bactéries dont la détection dans l'environnement témoigne généralement d'une pollution fécale récente.

Le Tableau 2 présente la comparaison des valeurs obtenues en termes d'analyses bactériologiques avec celles d'autres villes. Des concentrations moyennes des coliformes fécaux de $3,2.10^{5} \mathrm{UFC} / 100 \mathrm{ml}$ ont été relevées, tandis que celles des streptocoques fécaux sont de $3,08.10^{5} \mathrm{UFC} / 100 \mathrm{ml}$. Les concentrations sont du même ordre de grandeur que celles obtenues par Barro (2011) qui sont de $3,01.10^{5} \mathrm{UFC} / 100 \mathrm{ml}$ pour les Coliformes Fécaux (CF) tandis que lesStreptocoques Fécaux (SF) ont une teneur de $2,2.10^{6} \mathrm{UFC} / 100 \mathrm{ml}$, un peu plus élevée que la valeur obtenue à Zagtouli. Les teneurs relevées dans la présente étude sont également inférieures à celles rapportées par WHO (2006) dans différentes régions avec des teneurs dépassant quelque fois $10^{7} \mathrm{UFC} / 100$ mlen $\mathrm{CF}$. Cela confirme la grande variabilité des caractéristiques des boues d'un contexte à un autre.

Dans tous les cas, les analyses bactériologiques présentent un intérêt compte tenu des risques de contamination des eaux de surface (Gnagne et al., 2015) et des eaux de la nappe phréatique par les pathogènes (Natumanya et Okot-Okumu, 2015). A cet effet, les coliformes fécaux (CF) et les streptocoques fécaux (SF) indicateurs de pollution fécale permettant de guider le choix de la technologie de désinfection du lixiviat éventuellement.

\section{Conclusion}

La capitale du Burkina connaît une croissance exponentielle de sa population avec une augmentation des besoins dont ceux en ouvrage d'assainissement. Les ouvrages d'assainissement autonome sont les plus promus pour leur accessibilité. La gestion des sous-produits de ce type d'assainissement est une nécessité pour réduire l'incidence de leurs impacts sur la santé et sur l'environnement. Les 
analyses physicochimiques ainsi que les analyses bactériologiques montrent que les boues qui sont dépotées à la station de Zagtouli sont riches en matière organique, en nutriments $\mathrm{NH}_{4}, \mathrm{NO}_{3}, \mathrm{PO}_{4}$ mais également en bactéries pathogènes.

Il importe de respecter certaines normes pour réduire les risques de transmission de maladies, en évitant leur déversement dans la nature et leur utilisation en agriculture sans traitement préalable.

A l'issue de ce travail, au regard de la consistance et des caractéristiques chimiques et microbiologiques des boues, il peut être envisagé la possibilité de les traiter avantageusement par d'autres technologies rustiques telles que les lits plantés qui favorisent l'infiltration du lixiviat et une déshydratation plus rapide, ce d'autant plus que la capacité des deux stations est déjà dépassée eu égard au besoin réel de la ville.

\section{CONFLIT D'INTERETS}

Les auteurs ne déclarent aucun conflit d'intérêts en lien avec le présent article.

\section{CONTRIBUTIONS DES AUTEURS}

ES et MK ont contribué à la rédaction du manuscrit. OP et OY ont effectué les sorties de terrains et analyses au laboratoire. $\mathrm{PJ}$ et LB sont les universitaires encadreurs qui ont contribué par leurs soutiens, conseils et corrections.

\section{REFERENCES}

Barro R. 2011. Contribution à la mise en place d'une station de traitement des matières de vidange par lits de séchage plantés de Echinochloapyramidalis à Ouagadougou. Mémoire de Master II en Eau et Assainissement 2iE, p. 63.

Bricha S, Ounine K, Oulkheir S, El Haloui N, Attarassi B. 2007. Etude de la qualité physicochimique et bactériologique de la nappe phréatique M'nasra (Maroc). Afrique SCIENCE, 03(3): 391- 404.

Burkina Faso (2015). Décret $\mathrm{n}^{0}$ 20151205/PRESRANS/PM/MERH/MEF/MA RHASA/MS/MRA/MICA portant
Normes et conditions de déversements des eaux usées. Ouagadougou, P. 10.

Coulibaly L, Diomandé D, Coulibaly A, Gourène G. 2004. Utilisation des ressources en eaux, assainissement et risques sanitaires dans les quartiers précaires de la commune de Port-bouët (Abidjan; Cote d'ivoire). VertigO - La Revue en Sciences de l'Environnement. 5(3): 1-11.

Defo C, Fonkou T, Mabou PB, Nana P, Manjeli Y. 2015. Collecte et évacuation des boues de vidange dans la ville de Bafoussam, Cameroun (Afrique centrale) [VertigO] La Revue Electronique en Sciences de l'Environnement, 15(1).

Degrémont. 2005. Memento Technique de l'eau (Dixième édition, Tome 1), 785 p.

Gnagne YA, Yapo BO, Meite L, Kouamé VK, Gadji AA, Mambo V, Houenoul P. 2015. Caractérisation physico-chimique et bactériologique des eaux usées brutes du réseau d'égout de la ville d'Abidjan. Int. $J$. Biol. Chem. Sci., 9(2): 1082-1093.

Josse RG, Toklo RM, Dossou-Yovo P, Fatombi JK, Senou SF, Topanou N. 2016. Corrélation entre les résultats physico-chimiques et microbiologiques des lixiviats du lieu d'enfouissement sanitaire (LES) de Ouèssè/Ouidah et ceux des eaux souterraines et superficielles du milieu. Int. J. Biol. Chem. Sci., 10(2): 875-883.

Kengne N. I. M. 2006. Potentials of sludge drying beds vegetated with Cyperus papyrus L. and Echinochloapyramidalis (Lam.) Hitchc. \& Chase for faecal sludge treatment in tropical regions. $\mathrm{PhD}$ thesis, University of Yaounde I, Faculty of Science, Yaounde, p. 99.

Koné M. 2011. Infiltration-percolation sur sable et sur fibres de coco, filtres plantes et épuration d'eaux usées domestiques à dominance agroalimentaire sous climat tropical sec : cas des eaux résiduaires urbaines de Ouagadougou, Burkina Faso. Thèse unique, Université Claude Bernard Lyon 1, Lyon, p. 224. 
Koné M, Bonou L, Koulidiati J, Joly P, Sodré S, Bouvet Y. 2012. Traitement d'eaux usées urbaines par infiltration percolation sur sable et sur substrat de coco après un bassin anaérobie de lagunage sous climat tropical. Revue des Sciences de l'Eau 25(2): 139-151.

Koné D, Strauss M. 2004. Low-cost options for treating faecal sludges (FS) in developing countries; challenges and performance; Eawag/Sandec. p. 9

Koottatep T, Surinkul N, Polprasert C, Kamal ASM, Koné D, Montangero A, Heins U, Strauss M. 2005. Treatment of septage in constructed wetlands in tropical climate: lessons learnt from seven years of operation. Wat. Sci. \&Techn., 51(9): 119126.

Mahamane I. 2011. Contribution à la gestion durable des boues de vidange de la ville de Ouagadougou : Caractérisation des boues et évaluation du dimensionnement des STBV de Kossodo et Zagtouli. Mémoire de Master spécialisé 2iE, Ouagadougou, p. 68.

Natumanya R, Okot-Okumu J. 2015. Evaluating coagulant activity of locally available Syzygium cumini, Artocarpus heterophyllus and Moringa oleifera for treatment of community drinking water, Uganda. Int. J. Biol. Chem. Sci., 9(6): 2535-2554.

Nyenje PM, Foppen JW, Uhlenbrook S, Kulabako R, Muwanga A. 2010. Eutrophication and nutrient release in urban areas of sub-Saharan Africa. Science of the Total Environment, 408: 447-455.
OMS-UNICEF, 2010. Progrès en matière d'assainissement et d'alimentation en eau. OMS-UNICEF.

Rajmohan N, Elango L. 2005 Nutrient chemistry of groundwater in an intensively irrigated region of southern India. Environ Geol., 47: 820-830.

Strauss M, Koné D. 2003. Applied Research on the Management of Sludges from On-Site SanitationSystems in Developing Countries: Rationale, Issues and Programme Overview. EAWAG/ SANDEC, $7 \mathrm{p}$.

Tapsoba FW, Keré FD, Diarra J, Barry A, Sawadogo-Lingani H, Dianou D, Dicko MH. 2016. Etude de l'évolution des éléments précurseurs d'eutrophisation des eaux du Barrage $\mathrm{n}^{\circ} 3$ de Ouagadougou, Burkina Faso. Int. J. Biol. Chem. Sci., 10(2): 846-859.

Tilley E, Lüthi C, Morel A, Zurbrügg C, Schertenleib R. 2008. Compendium of sanitation systems and technologies, Dübendorf, Switzerland, Eawag, P. 158.

UN/Département des affaires économiques et sociales des Nations Unies (DAES). 2008. Objectifs du Millénaire pour le développement, Rapport 2008 Nations Unies New York, p. 56

Walker M. 2008. Performance of the FSTP Rufisque and its Impact on the WSP. Intership report, EAWAG / SANDEC, Zürich, p. 36.

WHO. 2006. Guidelines for the safe use of wastewater, excreta and greywater/excreta and greywater use in agriculture (volume IV). WHO; p. 204. 